\title{
Breaking and permanent waves for the periodic $\mu$-Degasperis-Procesi equation with linear dispersion
}

Fei Guo ${ }^{1 *}$

"Correspondence:

guof@njnu.edu.cn

'School of Mathematical Sciences and Jiangsu Key Laboratory for NSLSCS, Nanjing Normal University, Nanjing, P.R. China

\begin{abstract}
Considered herein is the periodic $\mu$-Degasperis-Procesi equation, which is an evolution equation on the space of tensor densities over the Lie algebra of smooth vector fields. First two conditions on the initial data that lead to breaking waves in finite time are formulated. The first breaking-wave result relies on the refined analysis on the evolution of the Lyapunov function $V(t)=\int_{\mathbb{S}} u_{x}^{3}(t, x) d x$; while the second result is based on the delicate comparison of the evolution of the solution $u$ and its gradient $u_{x}$. Second the existence of permanent waves is obtained by using an 'invariant' property of the momentum. Last the blow-up rate of breaking wave is determined by the argument of Constantin and Escher's well-known result on the evolution of the minimum of the gradient of the solution $u$.
\end{abstract}

MSC: 35B10; 35B44; 35G55; 35Q35

Keywords: Breaking wave; Permanent wave; Wave-breaking phenomena; Integrable system; $\mu$-Degasperis-Procesi equation; Blow-up rate

\section{Introduction}

The Degasperis-Procesi equation (DP)

$$
u_{t}-u_{t x x}+4 u u_{x}=3 u_{x} u_{x x}+u u_{x x x}
$$

was originally derived by Degasperis and Procesi $[18,19]$ using the method of asymptotic integrability up to third order as one of three equations in the family of third order dispersive PDE

$$
u_{t}-\alpha^{2} u_{x x t}+\gamma_{0} u_{x x x}+c_{0} u_{x}=\left(c_{1} u^{2}+c_{2} u_{x}^{2}+c_{3} u u_{x x}\right)_{x}
$$

The other two integrable equations in the family, after rescaling and applying a Galilean transformation, are the Korteweg-de Vries (KdV) equation

$$
u_{t}+u_{x x x}+u u_{x}=0
$$

(c) The Author(s) 2018. This article is distributed under the terms of the Creative Commons Attribution 4.0 International License (http://creativecommons.org/licenses/by/4.0/), which permits unrestricted use, distribution, and reproduction in any medium, provided you give appropriate credit to the original author(s) and the source, provide a link to the Creative Commons license, and indicate if changes were made. 
and the Camassa-Holm $(\mathrm{CH})$ shallow water equation $[5,16,23]$

$$
u_{t}-u_{t x x}+3 u u_{x}=2 u_{x} u_{x x}+u u_{x x x} .
$$

The $\mathrm{CH}$ and DP equations have attracted a lot of attention among the integrable systems and the PDE communities due to their two non-standard properties. The first most remarkable is the presence of "peakons" solutions $[5,19]$. These peakons are weak solutions in the distributional sense and shown to be stable in $[17,28]$. Another remarkable property is the occurrence of wave-breaking phenomena (i.e., a solution that remains bounded while its slope becomes unbounded in finite time) [11-14, 25, 29, 30].

It is noted that, for the $\mathrm{CH}$ equation, a solution after breaking can be continued as either a global conservative solution [2] or as a global dissipative solution [3], while for the DP equation shock waves possibly appear afterwards [32]. By now, there have been many papers devoted to the study of the DP equations, see, for example, $[1,6-9,15,21,22,26$, 28-31, 33].

The $\mathrm{CH}$ and DP equations are the cases $\lambda=2$ and $\lambda=3$, respectively, of the following family of equations:

$$
u_{t}-u_{t x x}+(\lambda+1) u u_{x}=\lambda u_{x} u_{x x x}+u u_{x x x}, \quad \lambda \in \mathbb{Z}
$$

Each equation in the family admits peakons [18] although only $\lambda=2$ and $\lambda=3$ are believed to be integrable [19]. Lenells, Misiolek, and Tiğlay [27] showed that each equation in the corresponding $\mu$-version of the family (1.4) given by

$$
\mu\left(u_{t}\right)-u_{t x x}+\lambda \mu(u) u_{x}=\lambda u_{x} u_{x x x}+u u_{x x x}, \quad \lambda \in \mathbb{Z}
$$

also admits peakon solutions, in which $\mu(u)=\int_{\mathbb{S}} u(t, x) d x$ is its mean and $\mu\left(u_{t}\right)$ has the similar meaning. The choices $\lambda=2$ and $\lambda=3$ yield the $\mu$-Camassa-Holm and $\mu$ Degasperis-Procesi ( $\mu$-DP) equations, respectively.

The $\mu$-DP equation can be formally described as an evolution equation on the space of tensor densities over the Lie algebra of smooth vector fields on the circle $\mathbb{S}$. As mentioned in [27], such geometric interpretation is not completely satisfactory. Recently, Escher, Kohlmann, and Kolev [20] verified that the periodic $\mu$-DP equation describes the geodesic flow of a right-invariant affine connection on the Frechet Lie group Diff ${ }^{\infty}(\mathbb{S})$ of all smooth and orientation-preserving diffeomorphisms of the circle $\mathbb{S}$. The $\mu$-DP equation admits the Lax pair

$$
\begin{aligned}
& \Psi_{x x x}=-\xi m \Psi, \\
& \Psi_{t}=-\frac{1}{\xi} \Psi_{x x}-u \Psi_{x}+u_{x} \Psi,
\end{aligned}
$$

where $\xi \in \mathbb{C}$ is a spectral parameter and $m=\mu(u)-u_{x x}$. Moreover, the $\mu$-DP equation also has a bi-Hamiltonian structure and an infinite hierarchy of conservation laws, therefore it is formally integrable.

It is easy to check that the $\mu$-DP equation is reversible, i.e., invariant under transformation $u \mapsto-u, t \mapsto-t$. However, it is not Galilean invariant, i.e., not invariant under 
$u \mapsto u+\kappa, t \mapsto t, x \mapsto x+\kappa t$. It lies in a family of equations parameterized by the critical shallow water speed $\kappa \in \mathbb{R}$ of the Galilean frame, i.e., the following equation:

$$
\left\{\begin{array}{l}
\mu\left(u_{t}\right)-u_{x x t}+3 \mu(u) u_{x}-3 u_{x} u_{x x}-u u_{x x x}+2 \kappa u_{x}=0, \quad t>0, x \in \mathbb{R} \\
u(0, x)=u_{0}(x), \quad x \in \mathbb{R}, \\
u(t, x+1)=u(t, x), \quad t \geq 0, x \in \mathbb{R} .
\end{array}\right.
$$

If $\kappa=0$ and $\mu(u)=0$, then Eq. (1.6) reduces to the short wave limit of the DegasperisProcesi equation [19] or the $\mu$-Burgers equation [27].

The goal of the present paper is to study the initial-value problem of the periodic Eq. (1.6) in the Sobolev space $H^{s}(\mathbb{S})$, where $\mathbb{S}=\mathbb{R} / \mathbb{Z}$. The Cauchy problem associated with Eq. (1.6) in the limiting case $\kappa=0$ was studied in [24,27]. We consider here the case $\kappa \neq 0$. Compared with the limiting case $\kappa=0$ (see [24, 27]), the difficulty we encounter is that the quantity $\mu(u)-u_{x x}$ cannot be conserved along the trajectory of the solution (cf., for example, the equation below (5.17) in [27]); as a replacement, we prove a similar conservation law (see Lemma 2.3). Based on this result, we can show that Eq. (1.6) has periodic waves that propagate at all times, as well as periodic waves that break in finite time, in the sense that the solution $u \in\left([0, T), H^{s}(\mathbb{S})\right), s>\frac{3}{2}$ with finite time $T<\infty$ satisfying $\liminf \operatorname{inT}_{t}\left(\inf _{x \in \mathbb{S}} u_{x}(t, x)\right)=-\infty$.

To analyze the breaking weaves, we provide two different approaches. The first one is based on a refined analysis on the evolution of the Lyapunov function $V(t)=\int_{\mathbb{S}} u_{x}^{3}(t, x) d x$, where some conserved quantities are involved (Theorem 3.1). The second one is based on the delicate comparison of the evolution of the solution $u$ and its gradient $u_{x}$. To our best knowledge, this approach was inspired by Constantin and Escher [11, 14]. We will use a continuous family of diffeomorphisms of the line associated with Eq. (1.6) to show that, for some "large" enough initial data, the corresponding solutions break in finite time. More precisely, by using an a priori estimate for the $L^{\infty}$-norm of the solution (Lemma 2.4), we find that the slope of the solution approaches infinitely in finite time or infinite time much faster than the solution itself even if it is unbounded. As a result, this leads to wave breaking (Theorem 3.2).

By virtue of an "invariant" property (Lemma 2.3), we can prove that if the initial data does not change its sign (Theorem 4.1), or its some Sobolev norm is appropriately "small" (Theorem 4.2), then the waves will exist permanently in time. In Constantin and Escher's pioneering paper [13], they proved that the "blow-up" quantity $m(t):=\inf _{x \in \mathbb{S}}\left[u_{x}(x, t)\right]$ is almost everywhere differential and the infimum can be obtained at points relying on the variable $t$. By this argument, we will determine the blow-up rate of the solutions (Theorem 5.1).

Throughout the paper, we identify all spaces of periodic functions with function spaces over $\mathbb{S}=\mathbb{R} / \mathbb{Z}$, and for simplicity we drop $\mathbb{S}$ in our notation.

\section{Preliminaries}

The initial-value problem (1.6) can be rewritten as

$$
\left\{\begin{array}{l}
u_{t}+u u_{x}+A^{-1} \partial_{x}\left(3 \mu_{0} u+2 \kappa u\right)=0, \quad t>0, x \in \mathbb{R} \\
u(0, x)=u_{0}(x), \quad x \in \mathbb{R} \\
u(t, x+1)=u(t, x), \quad t \geq 0, x \in \mathbb{R}
\end{array}\right.
$$


where $\mu_{0}$ is defined by (2.8), $A=\mu-\partial_{x}^{2}$ is an isomorphism between $H^{s}$ and $H^{s-2}$ with the inverse $v=A^{-1} w$ given explicitly by

$$
\begin{aligned}
v(x)= & \left(\frac{x^{2}}{2}-\frac{x}{2}+\frac{13}{12}\right) \mu(w)+\left(x-\frac{1}{2}\right) \int_{0}^{1} \int_{0}^{y} w(s) d s d y \\
& -\int_{0}^{x} \int_{0}^{y} w(s) d s d y+\int_{0}^{1} \int_{0}^{y} \int_{0}^{s} w(r) d r d s d y .
\end{aligned}
$$

Since $A^{-1}$ and $\partial_{x}$ commute, the following identities hold:

$$
A^{-1} \partial_{x} w(x)=\left(x-\frac{1}{2}\right) \int_{0}^{1} w(x) d x-\int_{0}^{x} w(y) d y+\int_{0}^{1} \int_{0}^{x} w(y) d y d x
$$

and

$$
A^{-1} \partial_{x}^{2} w(x)=-w(x)+\int_{0}^{1} w(x) d x .
$$

With $m=\left(\mu-\partial_{x}^{2}\right) u$, Eq. (1.6) can also be rewritten as

$$
m_{t}+u m_{x}+3 m u_{x}+2 \kappa u_{x}=0 .
$$

This will be used in the proof of the second breaking result (Theorem 3.2).

In the following, we establish some a priori estimates for Eq. (2.1). It is easy to verify that the first two conserved quantities for (2.1) are

$$
H_{0}=\int_{\mathbb{S}} m d x=\int_{\mathbb{S}}\left(\mu(u)-u_{x x}\right) d x=\mu(u)
$$

and

$$
H_{1}=\frac{1}{2} \int_{\mathbb{S}} u^{2} d x
$$

Let

$$
\mu_{0}=\mu(u(0))=\int_{\mathbb{S}} u(t, x) d x
$$

and

$$
\mu_{2}=\left(\int_{\mathbb{S}} u^{2}(0, x) d x\right)^{\frac{1}{2}}=\left(\int_{\mathbb{S}} u^{2}(t, x) d x\right)^{\frac{1}{2}} .
$$

Then $\mu_{0}$ and $\mu_{2}$ are constants and independent of time $t$ due to (2.6) and (2.7).

Lemma 2.1 ([10]) If $\in H^{3}$ is such that $\int_{\mathbb{S}} f(x) d x=a_{0} / 2$, then for every $\varepsilon>0$, we have

$$
\max _{x \in \mathbb{S}} f^{2}(x) \leq \frac{\varepsilon+2}{24} \int_{\mathbb{S}} f_{x}^{2}(x) d x+\frac{\varepsilon+2}{4 \varepsilon} a_{0}^{2}
$$


Remark 2.1 Since $H^{3}$ is dense in $H^{1}$, Lemma 2.1 also holds for every $f \in H^{1}$. Moreover, if $\int_{\mathbb{S}} f(x) d x=0$, from the deduction of this lemma we arrive at the following inequality:

$$
\max _{x \in \mathbb{S}} f^{2}(x) \leq \frac{1}{12} \int_{\mathbb{S}} f_{x}^{2}(x) d x, \quad f \in H^{1}
$$

Lemma 2.2 ([4]) For every $f(x) \in H^{1}(a, b)$ periodic and with zero average, i.e., $\int_{a}^{b} f(x) d x=$ 0 , we have

$$
\int_{a}^{b} f^{2}(x) d x \leq\left(\frac{b-a}{2 \pi}\right)^{2} \int_{a}^{b}\left|f_{x}(x)\right|^{2} d x
$$

and equality holds if and only if

$$
f(x)=A \cos \left(\frac{2 \pi x}{b-a}\right)+B \sin \left(\frac{2 \pi x}{b-a}\right) .
$$

The following proposition concerns the local well-posedness of (2.1), which was obtained in [27] (up to a slight modification, so the proof is omitted here).

Proposition 2.1 Let $u_{0} \in H^{s}, s>\frac{3}{2}$. Then there exist a maximal $T=T\left(u_{0}, \kappa\right)>0$ and $a$ unique solution $u$ to (2.1) such that

$$
u=u\left(\cdot, u_{0}\right) \in C\left([0, T), H^{s}\right) \cap C^{1}\left([0, T), H^{s-1}\right) .
$$

Moreover, the solution depends continuously on the initial data, that is, the mapping $u_{0} \mapsto$ $u\left(\cdot, u_{0}\right): H^{s} \rightarrow C\left([0, T), H^{s}\right) \cap C^{1}\left([0, T), H^{s-1}\right)$ is continuous.

Proposition 2.2 The maximal $T$ in Proposition 2.1 can be chosen independent of $s$ in the following sense. If $u=u\left(\cdot, u_{0}\right) \in C\left([0, T), H^{s}\right) \cap C^{1}\left([0, T), H^{s-1}\right)$ and $u_{0} \in H^{s^{\prime}}$ for some $s^{\prime} \neq s, s^{\prime}>\frac{3}{2}$, then $u \in C\left([0, T), H^{s^{\prime}}\right) \cap C^{1}\left([0, T), H^{s^{\prime}-1}\right)$ and with the same $T$. In particular, if $u_{0} \in H^{\infty}=\bigcap_{s \geq 0} H^{s}$, then $u \in C\left([0, T), H^{\infty}\right)$.

We now introduce Lagrangian coordinates for (2.1) in the following way. Assume that $u$ is the solution of (2.1). Given $x \in \mathbb{R}$, we associate with $u$ the following Cauchy problem:

$$
\begin{cases}q_{t}=u(t, q), & t \in[0, T), \\ q(0, x)=x, & x \in \mathbb{R} .\end{cases}
$$

Applying classical results in the theory of ordinary differential equations, we have the following properties of $q$ which are crucial in the proof of permanent waves.

Lemma 2.3 Let $u_{0} \in H^{s}, s>\frac{3}{2}$, and let $T>0$ be the maximal existence time of the corresponding solution $u$ to (2.1). Then (2.14) has a unique solution $q \in C^{1}([0, T) \times \mathbb{R}, \mathbb{R})$ such that the map $q(t, \cdot)$ is an increasing diffeomorphism of $\mathbb{R}$ with

$$
q_{x}(t, x)=\exp \left(\int_{0}^{t} u_{x}(s, q(s, x)) d s\right)>0, \quad \forall(t, x) \in[0, T) \times \mathbb{R} .
$$


Furthermore, setting $m=\mu(u)-u_{x x}$, we have

$$
\left(m(t, q(t, x))+\frac{2}{3} \kappa\right) q_{x}^{3}(t, x)=m_{0}(x)+\frac{2}{3} \kappa, \quad \forall(t, x) \in[0, T) \times \mathbb{R}
$$

Proof Since $u \in C^{1}\left([0, T), H^{s-1}\right)$ and $H^{s} \hookrightarrow C^{1}(\mathbb{S})$, we see that both functions $u(t, x)$ and $u_{x}(t, x)$ are bounded, Lipschitz in the space variable $x$, and of class $C^{1}$ in time. Therefore, for fixed $x \in \mathbb{R},(2.14)$ is an ordinary differential equation. Then, by the well-known classical results in the theory of ordinary differential equations, $(2.14)$ has a unique solution $q(t, x) \in$ $C^{1}([0, T) \times \mathbb{R}, \mathbb{R})$.

Differentiating (2.14) with respect to $x$ yields

$$
\left\{\begin{array}{l}
\frac{d}{d t} q_{x}=u_{x}(t, q) q_{x}, \quad t \in[0, T) \\
q_{x}(0, x)=1, \quad x \in \mathbb{R} .
\end{array}\right.
$$

The solution is given by

$$
q_{x}(t, x)=\exp \left(\int_{0}^{t} u_{x}(s, q(s, x)) d s\right)>0, \quad(t, x) \in[0, T) \times \mathbb{R} .
$$

For every $T^{\prime}<T$, it follows from the Sobolev embedding theorem that

$$
\sup _{(s, x) \in\left[0, T^{\prime}\right) \times \mathbb{R}}\left|u_{x}(s, x)\right|<\infty
$$

We infer from (2.16) that there exists a constant $K>0$ such that $q_{x}(t, x) \geq e^{-K t},(t, x) \in$ $\left[0, T^{\prime}\right) \times \mathbb{R}$, which implies that the map $q(t, \cdot)$ is an increasing diffeomorphism of $\mathbb{R}$ before blow-up.

On the other hand, using (2.5) and (2.14), we have

$$
\begin{aligned}
\frac{d}{d t}\left(m(t, q(t, x))+\frac{2}{3} \kappa\right) q_{x}^{3}(t, x) & =\left(m_{t}+m_{x} q_{t}\right) q_{x}^{3}+3\left(m+\frac{2}{3} \kappa\right) q_{x}^{2} q_{x t} \\
& =q_{x}^{3}\left(m_{t}+m_{x} u+3 m u_{x}+2 \kappa u_{x}\right)=0 .
\end{aligned}
$$

So,

$$
\left(m(t, q(t, x))+\frac{2}{3} \kappa\right) q_{x}^{3}(t, x)=m_{0}(x)+\frac{2}{3} \kappa, \quad \forall(t, x) \in[0, T) \times \mathbb{R}
$$

Remark 2.2 Lemma 2.3 shows that if $m_{0}(x)+\frac{2}{3} \kappa=\mu\left(u_{0}\right)-u_{0 x x}+\frac{2}{3} \kappa$ does not change sign, then $m(t)+\frac{2}{3} \kappa$ will not change sign as long as $m(t)$ exists.

Remark 2.3 Since $q(t, \cdot): \mathbb{R} \rightarrow \mathbb{R}$ is a diffeomorphism of the line for every $t \in[0, T)$, the $L^{\infty}$-norm of any function $u(t, \cdot) \in L^{\infty}, t \in[0, T)$ is preserved under the family of diffeomorphisms $q(t, \cdot)$ with $t \in[0, T)$, that is,

$$
\|u(t, \cdot)\|_{L^{\infty}}=\|u(t, q(t, \cdot))\|_{L^{\infty}}, \quad t \in[0, T) .
$$


The next result shows that, when a solution of (2.1) breaks in finite time or exists permanently, however, the solution itself remains bounded while the slope of the solution approaches infinitely in finite or infinite time much faster than the solution itself, in finite time this is just the wave-breaking phenomena. Based on this result, we can formulate a breaking result (Theorem 3.2).

Lemma 2.4 Assume $u_{0} \in H^{s}, s>\frac{3}{2}$. Let $T$ be the maximal time of the solution $u(t, x)$ to (2.1). Then we have

$$
\|u(t, \cdot)\|_{L^{\infty}} \leq\left|3 \mu_{0}+2 \kappa\right|\left(\frac{1}{2}\left|\mu_{0}\right|+2 \mu_{2}\right) t+\left\|u_{0}\right\|_{L^{\infty}}, \quad \forall t \in[0, T) .
$$

Proof Since the existence time $T$ is independent of the choice of $s$ by Proposition 2.2, applying a simple density argument, we only need to consider the case $s=3$. Let $T$ be the maximal existence time of the solution $u(t, x)$ to the initial-value problem (2.1) with the initial data $u_{0} \in H^{3}$.

In view of (2.3), (2.8)-(2.9), we have

$$
\left|A^{-1} \partial_{x} u\right| \leq \frac{1}{2}\left|\mu_{0}\right|+2 \mu_{2}
$$

From (2.14) it follows that

$$
\frac{d u(t, q(t, x))}{d t}=u_{t}(t, q(t, x))+u_{x}(t, q(t, x)) \frac{d q(t, x)}{d t}=\left(u_{t}+u u_{x}\right)(t, q(t, x)) .
$$

Combining the above estimates yields

$$
-\left|3 \mu_{0}+2 \kappa\right|\left(\frac{1}{2}\left|\mu_{0}\right|+2 \mu_{2}\right) \leq \frac{d u(t, q(t, x))}{d t} \leq\left|3 \mu_{0}+2 \kappa\right|\left(\frac{1}{2}\left|\mu_{0}\right|+2 \mu_{2}\right) .
$$

Integrating gives

$$
-\left|3 \mu_{0}+2 \kappa\right|\left(\frac{1}{2}\left|\mu_{0}\right|+2 \mu_{2}\right) t+u_{0}(x) \leq u(t, q(t, x)) \leq\left|3 \mu_{0}+2 \kappa\right|\left(\frac{1}{2}\left|\mu_{0}\right|+2 \mu_{2}\right) t+u_{0}(x) .
$$

So

$$
|u(t, q(t, x))| \leq\|u(t, q(t, \cdot))\|_{L^{\infty}} \leq\left|3 \mu_{0}+2 \kappa\right|\left(\frac{1}{2}\left|\mu_{0}\right|+2 \mu_{2}\right) t+\left\|u_{0}\right\|_{L^{\infty}} .
$$

In view of the diffeomorphism property of $q(t, \cdot)$, we obtain

$$
|u(t, \cdot)| \leq\|u(t, q(t, \cdot))\|_{L^{\infty}} \leq\left|3 \mu_{0}+2 \kappa\right|\left(\frac{1}{2}\left|\mu_{0}\right|+2 \mu_{2}\right) t+\left\|u_{0}\right\|_{L^{\infty}} .
$$

Constantin and Escher proved that the "blow-up" quantity $m(t):=\inf _{x \in \mathbb{S}}\left[u_{x}(x, t)\right]$ is almost everywhere differential and the infimum can be obtained at points relying on the variable $t$ [13]. We will determine the blow-up rate of the solutions of (2.1) using this argument (Theorem 5.1). 
Lemma $2.5([12,13])$ Let $T>0$ and $u \in C^{1}\left([0, T) ; H^{2}\right)$. Then, for every $t \in[0, T)$, there exists at least one point $\xi(t) \in \mathbb{S}$ with

$$
\omega(t):=\inf _{x \in \mathbb{S}}\left[u_{x}(t, x)\right]=u_{x}(t, \xi(t))
$$

and the function $\omega(t)$ is almost everywhere differentiable on $(0, T)$ with

$$
\frac{d \omega}{d t}=u_{t x}(t, \xi(t)) \quad \text { a.e. on }(0, T) \text {. }
$$

\section{Breaking waves}

In this section, we derive some sufficient conditions for the breaking waves to the initialvalue problem (2.1). We first state the precise wave-breaking scenario for problem (2.1).

Proposition 3.1 ([24]) Let $u_{0} \in H^{s}, s>\frac{3}{2}$, and $u(t, x)$ be the solution of the initial-value problem (2.1) with lifespan $T$. Then $T$ is finite if and only if

$$
\liminf _{t \uparrow T}\left(\inf _{x \in \mathbb{S}} u_{x}(t, x)\right)=-\infty
$$

In what follows, we establish some sufficient conditions guaranteeing the development of singularities by means of the wave-breaking scenario. The first result is based on the delicate analysis on the evolution of the Lyapunov function $V(t)=\int_{\mathbb{S}} u_{x}^{3}(t, x) d x$.

Theorem 3.1 Let $u_{0} \in H^{s}, s>\frac{3}{2}, u_{0} \neq 0$, and $T>0$ be the maximal time of existence of the corresponding solution $u(t, x)$ to (2.1) with the initial data $u_{0}$. If

$$
\frac{\mu_{2}^{2}}{8 \pi^{2}\left|\mu_{0}\right|\left(\mu_{2}^{2}-\mu_{0}^{2}\right)}<\frac{4}{\left|9 \mu_{0}+6 \kappa\right|}<\infty
$$

where $\mu_{0}, \mu_{2}$ are defined by (2.8) and (2.9), respectively, then $u(t, x)$ must blow up in finite time $T>0$ with the estimate

$$
\begin{aligned}
0 & <T \\
& \leq \inf _{\alpha \in I}\left(\frac{6}{4-\left|9 \mu_{0}+6 \kappa\right| \alpha}+2 \alpha \frac{1+\left|\int_{\mathbb{S}} u_{0 x}^{3}(x) d x\right|}{8 \pi^{2} \alpha\left|\mu_{0}\right|\left|9 \mu_{0}+6 \kappa\right|\left(\mu_{2}^{2}-\mu_{0}^{2}\right)-\left|9 \mu_{0}+6 \kappa\right| \mu_{2}^{2}}\right),
\end{aligned}
$$

where $I=\left(\frac{\mu_{2}^{2}}{8 \pi^{2}\left|\mu_{0}\right|\left(\mu_{2}^{2}-\mu_{0}^{2}\right)}, \frac{4}{\left|9 \mu_{0}+6 \kappa\right|}\right)$.

Proof Again as discussed previously, it suffices to prove the theorem only with $s=3$. We argue by contradiction. Assume that the solution exists globally. Differentiating Eq. (2.1) with respect to $x$ yields

$$
u_{t x}+u_{x}^{2}+u u_{x x}+A^{-1} \partial_{x}^{2}\left(3 \mu_{0} u+2 \kappa u\right)=0 .
$$

By (2.4) we deduce that

$$
u_{t x}=-u_{x}^{2}-u u_{x x}+\left(3 \mu_{0}+2 \kappa\right) u-\left(3 \mu_{0}^{2}+2 \kappa \mu_{0}\right) .
$$


Multiplying the equation by $3 u_{x}^{2}$ and integrating on $\mathbb{S}$ with respect to $x$, after integration by parts we obtain

$$
\begin{aligned}
\frac{d}{d t} \int_{\mathbb{S}} u_{x}^{3} d x= & -3 \int_{\mathbb{S}} u_{x}^{4} d x-\int_{\mathbb{S}} 3 u u_{x}^{2} u_{x x} d x+\left(9 \mu_{0}+6 \kappa\right) \int_{\mathbb{S}} u u_{x}^{2} d x \\
& -\left(9 \mu_{0}^{2}+6 \kappa \mu_{0}\right) \int_{\mathbb{S}} u_{x}^{2} d x \\
= & -2 \int_{\mathbb{S}} u_{x}^{4} d x+\left(9 \mu_{0}+6 \kappa\right) \int_{\mathbb{S}} u u_{x}^{2} d x-\left(9 \mu_{0}^{2}+6 \kappa \mu_{0}\right) \int_{\mathbb{S}} u_{x}^{2} d x
\end{aligned}
$$

It follows from Lemma 2.3 that

$$
\int_{\mathbb{S}}\left(u-\mu_{0}\right)^{2} d x \leq \frac{1}{4 \pi^{2}} \int_{\mathbb{S}} u_{x}^{2} d x .
$$

Obviously

$$
\int_{\mathbb{S}}\left(u-\mu_{0}\right)^{2} d x=\int_{\mathbb{S}}\left(u^{2}-2 \mu_{0} u+\mu_{0}^{2}\right) d x=\mu_{2}^{2}-\mu_{0}^{2}
$$

So

$$
\int_{\mathbb{S}} u_{x}^{2} d x \geq 4 \pi^{2}\left(\mu_{2}^{2}-\mu_{0}^{2}\right)
$$

For any $\alpha>0$ (to be determined later), it is easy to see that

$$
\begin{aligned}
\int_{\mathbb{S}} u u_{x}^{2} d x & \leq\left(\int_{\mathbb{S}} u^{2} d x\right)^{\frac{1}{2}}\left(\int_{\mathbb{S}} u_{x}^{4} d x\right)^{\frac{1}{2}} \leq \frac{\alpha}{2} \int_{\mathbb{S}} u_{x}^{4} d x+\frac{1}{2 \alpha} \int_{\mathbb{S}} u^{2} d x \\
& =\frac{\alpha}{2} \int_{\mathbb{S}} u_{x}^{4} d x+\frac{1}{2 \alpha} \mu_{2}^{2} .
\end{aligned}
$$

Substituting (3.9)-(3.10) back into (3.6) gives

$$
\begin{aligned}
& \frac{d}{d t} \int_{\mathbb{S}} u_{x}^{3} d x \\
& \quad \leq\left(\frac{\left|9 \mu_{0}+6 \kappa\right|}{2} \alpha-2\right) \int_{\mathbb{S}} u_{x}^{4} d x+\left|9 \mu_{0}+6 \kappa\right|\left(\frac{\mu_{2}^{2}}{2 \alpha}-4 \pi^{2}\left|\mu_{0}\right|\left(\mu_{2}^{2}-\mu_{0}^{2}\right)\right) .
\end{aligned}
$$

Set

$$
\frac{\left|9 \mu_{0}+6 \kappa\right|}{2} \alpha-2<0,
$$

i.e., $\alpha<\frac{4}{\left|9 \mu_{0}+6 \kappa\right|}$. If $\alpha>0$ also satisfies

$$
\frac{\left|9 \mu_{0}+6 \kappa\right|}{2 \alpha} \mu_{2}^{2}-4 \pi^{2}\left|\mu_{0}\right|\left|9 \mu_{0}+6 \kappa\right|\left(\mu_{2}^{2}-\mu_{0}^{2}\right)<0,
$$

then one finds that

$$
\alpha>\frac{\mu_{2}^{2}}{8 \pi^{2}\left|\mu_{0}\right|\left(\mu_{2}^{2}-\mu_{0}^{2}\right)} .
$$


Assumption (3.2) implies

$$
\frac{\mu_{2}^{2}}{8 \pi^{2}\left|\mu_{0}\right|\left(\mu_{2}^{2}-\mu_{0}^{2}\right)}<\frac{4}{\left|9 \mu_{0}+6 \kappa\right|} .
$$

Let

$$
c_{1}=2-\frac{\left|9 \mu_{0}+6 \kappa\right|}{2} \alpha>0
$$

and

$$
c_{2}=4 \pi^{2}\left|\mu_{0}\right|\left|9 \mu_{0}+6 \kappa\right|\left(\mu_{2}^{2}-\mu_{0}^{2}\right)-\frac{\left|9 \mu_{0}+6 \kappa\right|}{2 \alpha} \mu_{2}^{2}>0 .
$$

Then (3.11) becomes

$$
\frac{d}{d t} \int_{\mathbb{S}} u_{x}^{3} d x \leq-c_{1} \int_{\mathbb{S}} u_{x}^{4} d x-c_{2} \leq-c_{1}\left(\int_{\mathbb{S}} u_{x}^{3} d x\right)^{\frac{4}{3}}-c_{2}
$$

For any $t \geq 0$, define $V(t)=\int_{\mathbb{S}} u_{x}^{3} d x$. Then (3.18) becomes

$$
\frac{d}{d t} V(t) \leq-c_{1}(V(t))^{\frac{4}{3}}-c_{2} \leq-c_{2}<0, \quad \forall t \geq 0
$$

This implies that $V(t)$ decreases strictly for all $t \geq 0$. Let $t_{1}=(1+|V(0)|) / c_{2}$. Integrating (3.19) yields

$$
V\left(t_{1}\right)=V(0)+\int_{0}^{t_{1}} V^{\prime}(t) d t \leq|V(0)|-c_{2} t_{1}=-1 .
$$

Again by (3.19), we have

$$
\frac{d}{d t} V(t) \leq-c_{1}(V(t))^{\frac{4}{3}}, \quad t \geq t_{1}
$$

this implies that

$$
-3 \frac{d}{d t}\left(\frac{1}{(V(t))^{\frac{1}{3}}}\right)=(V(t))^{-\frac{4}{3}} \frac{d}{d t} V(t) \leq-c_{1}, \quad \forall t \geq t_{1} .
$$

Integrating and noting $V\left(t_{1}\right) \leq-1$ leads to

$$
-\frac{1}{(V(t))^{\frac{1}{3}}}-1 \leq-\frac{1}{(V(t))^{\frac{1}{3}}}+\frac{1}{\left(V\left(t_{1}\right)\right)^{\frac{1}{3}}} \leq-\frac{c_{1}}{3}\left(t-t_{1}\right), \quad t \geq t_{1} .
$$

Thus

$$
\left(\frac{c_{1}}{3}\left(t-t_{1}\right)+\frac{1}{\left(V\left(t_{1}\right)\right)^{\frac{1}{3}}}\right)^{3} \leq \frac{1}{V(t)}<0, \quad \forall t \geq t_{1} .
$$

We can derive a contradiction from the above inequality as $t \geq t_{1}$ is large enough. 
We now give the second blow-up result. For any $\varepsilon>0$, let

$$
\begin{aligned}
& a=4\left(3 \mu_{0}+2 \kappa\right)^{2}\left(\frac{1}{2}\left|\mu_{0}\right|+2 \mu_{2}\right), \\
& b=4\left|3 \mu_{0}+2 \kappa\right|\left(\left\|u_{0}\right\|_{L^{\infty}}-\mu_{0}\right), \\
& d=-\ln ^{2}\left(1+\frac{2}{\varepsilon}\right), \\
& p=-\frac{b^{2}}{3 a^{2}}, \\
& q=\frac{2 b^{3}}{27 a^{3}}+\frac{d}{a}, \\
& T_{1}=-\frac{b}{3 a}+\sqrt[3]{-\frac{q}{2}+\sqrt{\left(\frac{q}{2}\right)^{2}+\left(\frac{p}{3}\right)^{3}}}+\sqrt[3]{-\frac{q}{2}-\sqrt{\left(\frac{q}{2}\right)^{2}+\left(\frac{p}{3}\right)^{3}}}
\end{aligned}
$$

and

$$
P(t)=\left(\frac{1}{4} a t+\frac{1}{4} b\right)^{\frac{1}{2}}
$$

Actually, it is easy to check that $T_{1}$ is the only positive solution of the equation $4 P^{2}(t) t^{2}+$ $d=0$.

Theorem 3.2 Let $\varepsilon>0$. Assume that the initial profile $u_{0} \in H^{s}, s>\frac{3}{2}$ has at some point $x_{0} \in \mathbb{S}$ such that

$$
u^{\prime}\left(x_{0}\right) \leq-(1+\varepsilon) P\left(T_{1}\right)
$$

Then the solution of (2.1) breaks and the maximal time of existence is estimated above by $T_{1}$.

Proof As discussed earlier, it suffices to consider the case $s=3$. Let $T>0$ be the maximal time of existence of the solution $u$ to (2.1) with the initial data $u_{0} \in H^{3}$.

Let $\omega(t)=u_{x}\left(t, q\left(t, x_{0}\right)\right)$, where $q\left(t, x_{0}\right)$ is the flow of $u\left(t, q\left(t, x_{0}\right)\right)$. Then

$$
\frac{d \omega(t)}{d t}=\left(u_{t x}+u_{x x} q_{t}\right)\left(t, q\left(t, x_{0}\right)\right)=\left(u_{t x}+u u_{x x}\right)\left(t, q\left(t, x_{0}\right)\right)
$$

Substituting $\left(t, q\left(t, x_{0}\right)\right)$ into (3.5) and using Lemma 2.5, we obtain

$$
\begin{aligned}
\frac{d \omega(t)}{d t} & =-\omega^{2}(t)+\left(3 \mu_{0}+2 \kappa\right)\left(u\left(t, q\left(t, x_{0}\right)\right)-\mu_{0}\right) \\
& \leq-\omega^{2}(t)+\left|3 \mu_{0}+2 \kappa\right|\left[\left|3 \mu_{0}+2 \kappa\right|\left(\frac{1}{2}\left|\mu_{0}\right|+2 \mu_{2}\right) t+\left\|u_{0}\right\|_{L^{\infty}}-\mu_{0}\right] \\
& :=-\omega^{2}(t)+P^{2}(t) .
\end{aligned}
$$


By the definitions of $P(t)$ and $T_{1}$, we have

$$
2 P\left(T_{1}\right) T_{1}-\ln \left(1+\frac{2}{\varepsilon}\right)=0
$$

and

$$
\omega(0)<-(1+\varepsilon) P\left(T_{1}\right) .
$$

This implies that

$$
0<\frac{\omega(0)-P\left(T_{1}\right)}{\omega(0)+P\left(T_{1}\right)}=1-\frac{2 P\left(T_{1}\right)}{\omega(0)+P\left(T_{1}\right)} \leq 1+\frac{2}{\varepsilon} .
$$

Combining this inequality with (3.35) gives

$$
\frac{1}{2 P\left(T_{1}\right)} \ln \frac{\omega(0)-P\left(T_{1}\right)}{\omega(0)+P\left(T_{1}\right)} \leq T_{1} .
$$

By (3.33) and the definitions of $T_{1}$ and $P\left(T_{1}\right)$, we have

$$
\frac{d \omega(t)}{d t} \leq-\omega^{2}(t)+P^{2}\left(T_{1}\right), \quad \forall t \in\left[0, T_{1}\right) \cap[0, T) .
$$

Note that $\omega(0)<-(1+\varepsilon) P\left(T_{1}\right)<-P\left(T_{1}\right)$ and (3.36) for all $t \in\left[0, T_{1}\right) \cap[0, T)$, we can prove that $\omega(t)<-P\left(T_{1}\right)$ by the argument of continuous deduction. To this end, since $\omega(t)$ is continuous on $\left[0, T_{1}\right)$, failure of the above inequality would ensure the existence of some $t_{0} \in\left[0, T_{1}\right)$ such that $P^{2}\left(T_{1}\right)<\omega^{2}(t)$ on $\left[0, t_{0}\right)$, while $P^{2}\left(T_{1}\right)=\omega^{2}\left(t_{0}\right)$. But then we would have by (3.38)

$$
\frac{d \omega(t)}{d t}<0 \quad \text { a.e. } t \in\left(0, t_{0}\right)
$$

Being locally Lipschitz, the function $\omega$ is absolutely continuous on $\left[0, t_{0}\right]$, and therefore an integration of the previous inequality would lead us to

$$
\omega\left(t_{0}\right) \leq m(0)<-P\left(T_{1}\right)
$$

which contradicts our assumption $P^{2}\left(T_{1}\right)=\omega^{2}\left(t_{0}\right)$.

Solving inequality (3.38) yields

$$
\frac{\omega(0)+P\left(T_{1}\right)}{\omega(0)-P\left(T_{1}\right)} e^{2 P\left(T_{1}\right) t}-1 \leq \frac{2 P\left(T_{1}\right)}{\omega(t)-P\left(T_{1}\right)} \leq 0 .
$$

In view of $0<\frac{\omega(0)+P\left(T_{1}\right)}{\omega(0)-P\left(T_{1}\right)}<1$, we deduce that there exists $T$ satisfying

$$
0<T<\frac{1}{2 P\left(T_{1}\right)} \ln \frac{\omega(0)+P\left(T_{1}\right)}{\omega(0)-P\left(T_{1}\right)} \leq T_{1}
$$

such that $\lim _{t \uparrow T} \omega(t)=-\infty$, which completes the proof of this theorem. 


\section{Permanent waves}

In this section, attention is now turned to specifying conditions under which the local solution to the initial-value problem (2.1) can be extended to a global one.

Theorem 4.1 If the initial potential value $m_{0} \in H^{1}(\mathbb{S})$ satisfies that $m_{0}+\frac{2}{3} \kappa$ does not change its sign, then the solution $u(t, x)$ to the initial-value problem (2.1) exists permanently in time.

Proof Let $T$ be the maximal time of existence of the solution $u$ to (2.1) with the initial data $u_{0}$ guaranteed by Proposition 2.2.

Assume $m_{0}+\frac{2}{3} \kappa \geq 0$, we prove that the solution $u(t, x)$ exists globally in time. Indeed, owing to Lemma 2.3 and Remark 2.2, we find $m(t)+\frac{2}{3} \kappa \geq 0$ on $[0, T) \times \mathbb{S}$. Given $t \in[0, T)$, by the periodicity in the $x$-variable, there is $\xi(t) \in(0,1)$ such that $u_{x}(t, \xi(t))=0$. Therefore, for $x \in[\xi(t), \xi(t)+1]$, we have

$$
\begin{aligned}
- & u_{x}(t, x) \\
& =-\int_{\xi(t)}^{x} \partial_{x}^{2} u(t, x) d x=\int_{\xi(t)}^{x}\left(m(t, x)+\frac{2}{3} \kappa\right) d x-\int_{\xi(t)}^{x}\left[\mu(u)+\frac{2}{3} \kappa\right] d x \\
& \leq \int_{\xi(t)}^{\xi(t)+1}\left(m(t, x)+\frac{2}{3} \kappa\right) d x-\left(\mu_{0}+\frac{2}{3} \kappa\right)(x-\xi(t)) \\
& =\left(\mu_{0}+\frac{2}{3} \kappa\right)(1-x+\xi(t)) \leq\left|\mu_{0}+\frac{2}{3} \kappa\right| .
\end{aligned}
$$

Similarly, if $m_{0}+\frac{2}{3} \kappa \leq 0$, then $m(t)+\frac{2}{3} \kappa \leq 0$ on $[0, T) \times \mathbb{S}$. Using the same notation as above, we find that

$$
\begin{aligned}
- & u_{x}(t, x) \\
& =-\int_{\xi(t)}^{x} \partial_{x}^{2} u(t, x) d x=\int_{\xi(t)}^{x}\left(m(t, x)+\frac{2}{3} \kappa\right) d x-\int_{\xi(t)}^{x}\left[\mu(u)+\frac{2}{3} \kappa\right] d x \\
& =-\left(\mu_{0}+\frac{2}{3} \kappa\right)(x-\xi(t)) \\
& \leq\left|\mu_{0}+\frac{2}{3} \kappa\right| .
\end{aligned}
$$

Combining (4.1) with (4.2), we deduce that $u$ exists permanently as a consequence of Proposition 3.1.

Theorem 4.2 If the initial profile $u_{0} \in H^{3}(\mathbb{S})$ is such that

$$
\left\|\partial_{\varkappa}^{3} u_{0}\right\|_{L^{2}} \leq 2 \sqrt{3}\left|u_{0}+\frac{2}{3} \kappa\right|
$$

then the wave of (2.1) exists permanently.

Proof Let $T$ be the maximal time of existence of the solution $u$ to (2.1) with initial data $u_{0}$ guaranteed by Proposition 2.2. 
By Remark 2.1 we get

$$
\max \left(\partial_{x}^{2} u_{0}\right)^{2} \leq \frac{1}{12} \int_{\mathbb{S}}\left(\partial_{x}^{3} u_{0}\right)^{2} d x
$$

so

$$
\left\|\partial_{x}^{2} u_{0}\right\|_{L^{\infty}} \leq \frac{\sqrt{3}}{6}\left\|\partial_{x}^{3} u_{0}\right\|_{L^{2}}
$$

If $\mu_{0}+\frac{2}{3} \kappa \geq 0$, it is inferred from (4.3) and (4.5) that

$$
m_{0}+\frac{2}{3} \kappa=\mu_{0}+\frac{2}{3} \kappa-\partial_{x}^{2} u_{0} \geq \mu_{0}+\frac{2}{3} \kappa-\frac{\sqrt{3}}{6}\left\|\partial_{x}^{3} u_{0}\right\|_{L^{2}} \geq 0
$$

Similarly, if $u_{0}+\frac{2}{3} \kappa \leq 0$, one obtains from (4.3) and (4.5) that

$$
m_{0}+\frac{2}{3} \kappa=\mu_{0}+\frac{2}{3} \kappa-\partial_{x}^{2} u_{0} \leq \mu_{0}+\frac{2}{3} \kappa+\frac{\sqrt{3}}{6}\left\|\partial_{x}^{3} u_{0}\right\|_{L^{2}} \leq 0 .
$$

Therefore, in view of Theorem 4.1, the proof of this theorem is completed.

\section{Blow-up rate}

Our attention is now turned to the problem of the blow-up rate of the slope to a breaking wave for the initial-value problem (2.1).

Theorem 5.1 Let $u(t, x)$ be the solution to the initial-value problem (2.1) with initial data $u_{0} \in H^{s}, s>\frac{3}{2}$. Let $T>0$ be the maximal time of existence of the solution $u(t, x)$. If $T<\infty$, we have

$$
\lim _{t \uparrow T}\left\{\inf _{x \in \mathbb{S}}\left[u_{x}(t, x)(T-t)\right]\right\}=-1
$$

while the solution remains uniformly bounded.

Proof Again we may assume $s=3$ to prove this theorem. The uniform boundedness of the solution can be easily obtained as a consequence of the a priori estimate in Lemma 2.4.

By Lemma 2.5, the function

$$
\omega(t):=\inf _{x \in \mathbb{R}}\left[u_{x}(t, x)\right]=u_{x}(t, \xi(t))
$$

is locally Lipschitz with $\omega(t)<0, t \in[0, T)$. Note that $u_{x x}(t, \xi(t))=0$ for a.e. $t \in[0, T)$. Then

$$
\omega^{\prime}(t)=-\omega^{2}(t)+\left(3 \mu_{0}+2 \kappa\right) u(t, \xi(t))-\left(3 \mu_{0}^{2}+2 \kappa \mu_{0}\right), \quad t \in[0, T)
$$


By Lemma 2.9 we obtain

$$
\begin{aligned}
& \left|\left(3 \mu_{0}+2 \kappa\right)\left[u(t, \xi(t))-\mu_{0}\right]\right| \\
& \quad \leq\left|3 \mu_{0}+2 \kappa\right|\left(\|u(t, \cdot)\|_{L^{\infty}}+\left|\mu_{0}\right|\right) \\
& \quad \leq\left|3 \mu_{0}+2 \kappa\right|\left[\left|3 \mu_{0}+2 \kappa\right|\left(\frac{1}{2}\left|\mu_{0}\right|+2 \mu_{2}\right) t+\left\|u_{0}\right\|_{L^{\infty}}+\left|\mu_{0}\right|\right], \quad t \in[0, T) \\
& \quad \triangleq N(t) .
\end{aligned}
$$

Then we have

$$
\omega^{\prime}(t) \leq-\omega^{2}(t)+N(T), \quad t \in[0, T)
$$

Now fix any $\varepsilon \in(0,1)$. In view of Proposition 3.1, there exists $t_{0} \in(0, T)$ such that $\omega\left(t_{0}\right)<$ $-\sqrt{N(T)+\frac{N(T)}{\varepsilon}}$. Notice that $\omega(t)$ is locally Lipschitz so that it is absolutely continuous on $[0, T)$. It then follows from $(5.5)$ that $\omega(t)$ is decreasing on $\left[t_{0}, T\right)$ and satisfies that

$$
\omega(t)<-\sqrt{N(T)+\frac{N(T)}{\varepsilon}}<-\sqrt{\frac{N(T)}{\varepsilon}}, \quad t \in\left[t_{0}, T\right) .
$$

Since $\omega(t)$ is decreasing on $\left[t_{0}, T\right)$, it follows that

$$
\lim _{t \uparrow T} \omega(t)=-\infty
$$

In view of (5.6), from (5.5) we deduce that

$$
1-\varepsilon \leq \frac{d}{d t}\left(\omega(t)^{-1}\right)=-\frac{\omega^{\prime}(t)}{\omega(t)^{2}} \leq 1+\varepsilon
$$

Integrating the above equation on $(t, T)$ with $t \in\left(t_{0}, T\right)$ and noticing that $\lim _{t \uparrow T} \omega(t)=-\infty$, we get

$$
(1-\varepsilon)(T-t) \leq-\frac{1}{\omega(t)} \leq(1+\varepsilon)(T-t) .
$$

Since $\varepsilon \in(0,1)$ is arbitrary, in view of the definition of $\omega(t)$, the above inequality implies the desired result of Theorem 5.1 .

\section{Acknowledgements}

The author is grateful for the constructive suggestions made by the referees.

\section{Funding}

This work is supported by the Priority Academic Program Development of Jiangsu Higher Education Institutions, the Natural Science Foundation of Jiangsu Province (BK20181381), the NSF of the Jiangsu Higher Education Institutions (17KJA110002) and Qing Lan Projects of Jiangsu Province and Nanjing Normarl University.

\section{Competing interests}

The author declares that they have no competing interests. 


\section{Publisher's Note}

Springer Nature remains neutral with regard to jurisdictional claims in published maps and institutional affiliations.

\section{Received: 12 July 2018 Accepted: 5 November 2018 Published online: 17 November 2018}

\section{References}

1. Brandolese, L.: A Liouville theorem for the Degasperis-Procesi equation. Ann. Sc. Norm. Super. Pisa, Cl. Sci. 16, 759-765 (2016)

2. Bressan, A., Constantin, A.: Global conservative solutions of the Camassa-Holm equation. Arch. Ration. Mech. Anal. $183,215-239(2007)$

3. Bressan, A., Constantin, A.: Global dissipative solutions of the Camassa-Holm equation. Anal. Appl. 5, 1-27 (2007)

4. Buttazo, G., Giaquina, M., Hildebrandt, S.: One-Dimensional Variational Problems: An Introduction. Clarendon, Oxford (1998)

5. Camassa, R., Holm, D.D.: An integrable shallow water equation with peaked solitons. Phys. Rev. Lett. 71, 1661-1664 (1993)

6. Chen, R.M., Guo, F., Liu, Y., Qu, C.Z:: Analysis on the blow-up of solutions to a class of integrable peakon equations. J. Funct. Anal. 270, 2343-2374 (2016)

7. Coclite, G.M., Karlsen, K.H.: On the well-posedness of the Degasperis-Procesi equation. J. Funct. Anal. 233, 60-91 (2006)

8. Coclite, G.M., Karlsen, K.H.: On the uniqueness of discontinuous solutions to the Degasperis-Procesi equation. J. Differ. Equ. 234, 142-160 (2007)

9. Coclite, G.M., Karlsen, K.H.: Bounded solutions for the Degasperis-Procesi equation. Boll. Unione Mat. Ital., B 1, 439-453 (2008)

10. Constantin, A.: On the blow-up of solutions of a periodic shallow water equation. J. Nonlinear Sci. 10, 391-399 (2000)

11. Constantin, A.: Existence of permanent and breaking waves for a shallow water equation: a geometric approach. Ann. Inst. Fourier (Grenoble) 50, 321-362 (2000)

12. Constantin, A.: Nonlinear Water Waves with Applications to Wave-Current Interactions and Tsunamis. CBMS-NSF Regional Conference Series in Applied Mathematics, vol. 81 (2011)

13. Constantin, A., Escher, J.: Wave breaking for nonlinear nonlocal shallow water equations. Acta Math. 181, 229-243 (1998)

14. Constantin, A., Escher, J.: Well-posedness, global existence and blowup phenomena for a periodic quasi-linear hyperbolic equation. Commun. Pure Appl. Math. 51, 475-504 (1998)

15. Constantin, A., Ivanov, R.: Dressing method for the Degasperis-Procesi equation. Stud. Appl. Math. 138, 205-226 (2017)

16. Constantin, A., Lannes, D.: The hydrodynamical relevance of the Camassa-Holm and Degasperis-Procesi equations. Arch. Ration. Mech. Anal. 192, 165-186 (2009)

17. Constantin, A., Strauss, W.A.: Stability of peakons. Commun. Pure Appl. Math. 53, 603-610 (2000)

18. Degasperis, A., Holm, D.D., Hone, A.N.W.: A new integrable equation with peakon solutions. Teor. Mat. Fiz. 133, $1463-1474$ (2002)

19. Degasperis, A., Procesi, M.: Asymptotic integrability. In: Degasperis, A., Gaeta, G. (eds.) Symmetry and Perturbation Theory, pp. 23-37. World Scientific, Singapore (1999)

20. Escher, J., Kohlmann, M., Kolev, B.: Geometric aspects of the periodic $\mu$-Dasperis-Procesi equation, parabolic problems. In: Progr. Nonlinear Differential Equations Appl., vol. 80, pp. 193-209. Birkhüser, Basel (2011)

21. Escher, J., Liu, Y., Yin, Z.Y.: Global weak solutions and blow-up structure for the Degasperis-Procesi equation. J. Funct. Anal. 241, 457-485 (2006)

22. Escher, J., Liu, Y., Yin, Z.Y.: Shock waves and blow-up phenomena for the periodic Degasperis-Procesi equation. Indiana Univ. Math. J. 56, 87-117 (2007)

23. Fokas, A.S., Fuchssteiner, B.: Symplectic structures, their Bäcklund transformations and hereditary symmetries. Physica D 4, 47-66 (1981/82)

24. Fu, Y., Liu, Y., Qu, C.Z:: On the blow-up structure for the generalized periodic Camassa-Holm and Degasperis-Procesi equations. J. Funct. Anal. 262, 3125-3158 (2012)

25. Guo, F:: On the curvature blow-up phenomena for the Fokas-Qiao-Xia-Li equation. J. Math. Anal. Appl. 450, 1275-1293 (2017)

26. Guo, F., Gao, H.J., Liu, Y.: Existence of permanent and breaking waves for the periodic Degasperis-Procesi equation with linear dispersion. J. Reine Angew. Math. 657, 199-223 (2011)

27. Lenells, J., Misiolek, G., Tiğlay, F.: Integrable evolution equations on spaces of tensor densities and their peakon solutions. Commun. Math. Phys. 299, 129-161 (2010)

28. Lin, Z.W., Liu, Y.: Stability of peakons for the Degasperis-Procesi equation. Commun. Pure Appl. Math. 62, 125-146 (2009)

29. Liu, Y., Yin, Z.Y.: Global existence and blow-up phenomena for the Degasperis-Procesi equation. Commun. Math. Phys. 267, 801-820 (2006)

30. Liu, Y., Yin, Z.Y.: On the blow-up phenomena for the Degasperis-Procesi equation. Int. Math. Res. Not. 2007, 117 (2007)

31. Lundmark, H.: Formation and dynamics of shock waves in the Degasperis-Procesi equation. J. Nonlinear Sci. 17, 169-198 (2007)

32. Lundmark, H., Szmigielski, J.: Multi-peakon solutions of the Degasperis-Procesi equation. Inverse Probl. 19, 1241-1245 (2003)

33. Matsuno, Y.: Multisoliton solutions of the Degasperis-Procesi equation and their peakon limit. Inverse Probl. 21, 1553-1570 (2005) 